\title{
HEMOAPP: APLICATIVO PARA MANEJO DO PACIENTE PORTADOR DE COAGULOPATIAS HEREDITÁRIAS.
}

\author{
CENTRO/NOVA IGUAÇU/RJ JULHO/2018
}

\author{
Juliana de Medeiros Matos - UERJ - drajumatos@gmail.com \\ Marcia Maria Pereira - UERJ - mmrendeiro@gmail.com
}

Tipo: Relato de Experiência Inovadora (EI)

Categoria: Métodos e Tecnologias

Setor Educacional: EDUCAÇÃO SUPERIOR

\begin{abstract}
RESUMO
O objetivo do presente estudo foi elaborar um aplicativo que auxiliasse o profissional de saúde no manejo do paciente portador de coagulopatias hereditárias, auxiliando na assistência e educação permanente desses profissionais. Este é um estudo descritivo do desenvolvimento experimental de um aplicativo. Foram criadas 44 telas, o aplicativo foi desenvolvido em 2 fases: elaboração do protótipo e pré-teste. O aplicativo desenvolvido para plataforma Android, utilizando a linguagem JAVAScript com framework Reactive-Native. O aplicativo foi dividido em 2 módulos: módulo Hemofilia e módulo Von Willebrand. Como resultado, o aplicativo foi projetado para a plataforma Android, observando os princípios de usabilidade e ergonomia. O aplicativo foi denominado "Hemoapp" e foram criadas 44 telas. O aplicativo para dispositivos móveis possibilita não só o suporte com evidências científicas para avaliação e classificação do paciente portador de coagulopatias hereditárias, como também a capacitação dos profissionais no cuidado, o que permite um gerenciamento de qualidade do atendimento desses pacientes na atenção primária, evitando deslocamentos até os Hemocentros. Após elaboração do aplicativo, realizou-se um pré-teste com profissionais de saúde da APS a fim de avaliar sua ergonomia, usabilidade e levantar possíveis modificações a serem realizadas no aplicativo e nos questionários de avaliação. Por meio do pré-teste pudemos verificar também a necessidade contínua de atualização profissional e a incorporação de novas tecnologias no cuidado, que acabam por facilitar e agilizar a assistência do paciente.
\end{abstract}

Palavras-chave: Doença de Von Willebrand. Hemofilia. Coagulopatias. Informática em Saúde

\section{AGRADECIMENTOS}

À MINHA QUERIDA MÃE, CLARA, POR ESTAR AO MEU LADO EM TODOS OS MOMENTOS DE MINHA VIDA E APOIAR INCONDICIONALMENTE 
Introdução

As coagulopatias hereditárias são doenças hemorrágicas resultantes da deficiência quantitativa e/ou qualitativa de um ou mais fatores de coagulação. Entre as coagulopatias, as mais comuns estão a hemofilia e a doença de Von Willebrand (DVW). (LORENZI, 2003). A hemofilia é uma doença hereditária ligada ao cromossomo X, caracterizada laboratorialmente pela deficiência dos fatores de coagulação VIII ou IX, nas hemofilias $A$ e $B$ respectivamente. $A$ hemofilia $A$ é mais prevalente que a hemofilia $B$, ocorrendo em cerca de 1:10.000-20.000 homens. (FEDERAÇÃO BRASILEIRA DE HEMOFILIA,2016)

As hemofilias caracterizam-se clinicamente por sangramentos prolongados, principalmente em musculatura profunda, em articulações e, após procedimentos cirúrgicos, principalmente na cavidade bucal. A DVW é outra coagulopatia de grande incidência populacional. É caracterizada pela deficiência qualitativa e/ou quantitativa do fator de Von Willebrand (FVW), que é uma proteína que facilita a adesão plaquetária ao endotélio. Ela é também carreadora do fator VIII no plasma, protegendo-o da destruição prematura por proteases plasmáticas. A DVW é mais comum das coagulopatias hereditárias, sendo mais comumente transmitida por herança autossômica dominante, podendo, entretanto, ter expressão fenotípica variável. A DVW é classificada em três tipos: 1, 2 e 3, sendo o tipo 2 subclassificado em 4 subtipos. (LORENZI, 2003), (PETROVITCH,2004)

No editorial Perfil das Coagulopatias hereditárias no Brasil, o Ministério da Saúde, tendo como fonte de informação o Sistema Hemovida Web Coagulopatias, divulga o quantitativo dos pacientes portadores desses tipos de alterações no país. Em 2010, o total de pacientes com coagulopatias hereditárias no Brasil atingiu o número de 16.076 pacientes, dos quais $8.369(52,06 \%)$ correspondem à hemofilia $A ; 1.609(10,01 \%)$, à hemofilia $B ; 4.451$ (27,69\%), à doença de Von Willebrand; e $1.647(10,25 \%)$, a outras coagulopatias hereditárias e aos demais transtornos hemorrágicos. Já em 2014, houve um aumento considerável, totalizando em 21.066 o número de pacientes com coagulopatias hereditárias no Brasil, dos quais 9.616 (45,65\%) correspondem à hemofilia $A ; 1.881$ (8,93\%), à hemofilia $B ; 6.544$ (31,06\%), à doença de von Willebrand; e $3.025(14,36 \%)$, a outras coagulopatias hereditárias e aos demais transtornos hemorrágicos. (BRASIL,2015b)

Devido ao grande número de brasileiros portadores de coagulopatias hereditárias e suas dificuldades em conseguir atendimento adequado e orientações em unidades de saúde que não sejam Hemocentros, acreditamos que o desenvolvimento de aplicativos 
para dispositivos móveis possibilitaria não só o suporte com evidências científicas para avaliação e classificação do paciente portador de coagulopatias hereditárias, como a capacitação dos profissionais no cuidado e orientações aos pacientes em relação ao seu tratamento. O presente artigo tem como apresentar o aplicativo "Hemoapp" desenvolvido durante o Mestrado em Telessaúde e Telemedicina da UERJ.

\section{Metodologia}

Trata-se de um estudo descritivo de desenvolvimento experimental de um aplicativo, com abordagem quantitativa, desenvolvido na plataforma Android. A plataforma surgiu de uma parceria entre a Google e a Open Handset Alliance (OHA), um grupo de 84 empresas que se uniram para inovar e acelerar o número de consumidores de dispositivos móveis oferecendo uma experiência rica e de preço mais acessível. Além de inúmeras funcionalidades, a plataforma Android é completamente livre e de código aberto, o que torna possível utilizar gratuitamente o sistema operacional, além de poder personaliza-lo, sendo esses os principais motivos para a escolha da plataforma Android para o desenvolvimento deste estudo.

Para construção do sistema foi utilizado para desenvolvimento e gerenciamento do projeto as metodologias ágeis. As metodologias ágeis para desenvolvimento de software são uma resposta às chamadas metodologias pesadas ou tradicionais. O que as diferencia das metodologias tradicionais são o enfoque e os valores. Uma característica das metodologias ágeis é que elas são adaptativas ao invés de serem preditivas. Com isso, ao invés de procurar analisar previamente tudo o que pode acontecer no decorrer do desenvolvimento elas acabam se adaptando a novos fatores decorrentes do desenvolvimento do projeto. (COCKBURN,2001)

A linguagem utilizada para desenvolvimento do aplicativo foi a linguagem JavaScript com framework Reactive-Native. JavaScript é uma linguagem de programação interpretada. Originalmente foi implementada como parte dos navegadores web para que scripts pudessem ser executados do lado do cliente e interagissem com o usuário sem a necessidade deste script passar pelo servidor, controlando o navegador, realizando comunicação assíncrona e alterando o conteúdo do documento exibido. (FLANAGAN,2002)

\section{Resultados}


Foram elaboradas 44 telas que compõem as variáveis necessárias para auxílio ao atendimento do paciente portador de coagulopatias pelo profissional. A figura 1 ilustra 0 ícone do aplicativo na tela menu do Smartphone. Ao acessar o aplicativo (app) o usuário será direcionado para preencher o formulário de cadastro (figura 2), irá preencher com seus dados para prosseguir na utilização do aplicativo. Os dados solicitados servirão para que no futuro a pesquisadora faça um levantamento sobre as profissões e os estados que mais utilizam o app.

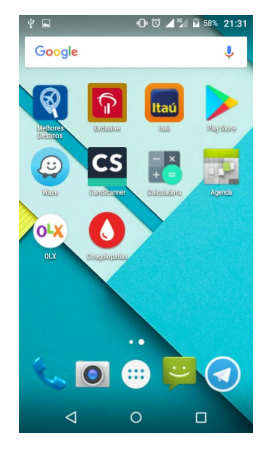

Figura 1 - Tela de menu do smartphone

Fonte: Próprio autor, 2017

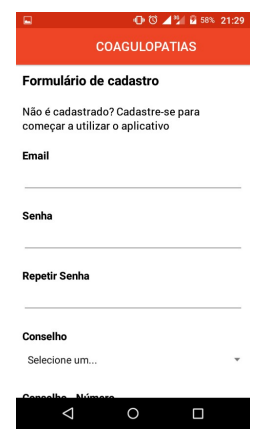

Figura 2 - Formulário de cadastro

Fonte: Próprio autor, 2017

Ao finalizar o cadastro o usuário será direcionado a uma página com o termo de aceite, que inclui os Manuais do Ministério da Saúde que foram utilizadas como base científica para a confecção e as categorias profissionais a que se destinam o app, o profissional poderá selecionar na caixa de diálogo a opção de não exibir essa mensagem novamente, caso não selecione a opção sempre que abrir o aplicativo esse mensagem aparecerá, até que selecione a opção. O profissional visualizará as duas coagulopatias hereditárias que foram selecionadas para compor o app: Hemofilia e Doença de Von Willebrand (figura 3). 


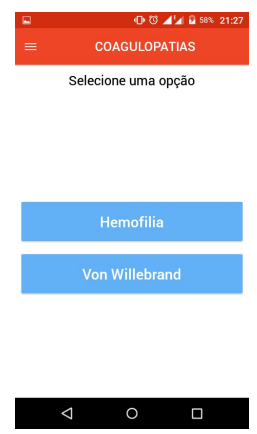

Figura 3- Página inicial do aplicativo

Fonte: próprio autor, 2017

Para acessar o menu de opções o usuário deve clicar sobre o botão localizado no canto superior direito da tela. No menu o profissional encontrará as opções de retornar ao início, acessar as configurações, saber mais sobre o aplicativo ou poderá enviar sugestões para os autores. Ao acessar as configurações o profissional poderá alterar seus dados cadastrais. Na opção Sobre terá acessos aos dados de desenvolvimento do app, como autor, orientador, desenvolvedor e bibliografia utilizada. Na aba Sugestões o usuário poderá enviar um e-mail aos autores com sugestões para aprimoramento do aplicativo, bem como dúvidas e críticas.

Se optar por acessar o módulo de Hemofilia o profissional será direcionado para a tela do menu referente ao módulo. O usuário poderá acessar a definição da doença, saber mais sobre o diagnóstico da Hemofilia, classificação e tratamento dos pacientes. Se optar por selecionar o módulo Von Willebrand o usuário encontrará um menu igual ao do módulo de Hemofilia. O usuário poderá acessar a definição da doença, saber mais sobre o diagnóstico da Doença de Von Willebrand, na opção classificação e tratamento dos pacientes o profissional terá acesso a uma tela com os subtipos da doença e a opção dos tratamentos. Assim que clicar em cada subtipo o profissional acessará a definição de cada um deles.

Após elaboração do aplicativo, realizou-se um pré-teste com profissionais de saúde da APS a fim de avaliar sua ergonomia, usabilidade e levantar possíveis modificações a serem realizadas no aplicativo e nos questionários de avaliação. Por meio do pré-teste pudemos verificar também a necessidade contínua de atualização profissional e a incorporação de novas tecnologias no cuidado, que acabam por facilitar e agilizar a assistência do paciente. O pré-teste foi realizado com 20 profissionais de saúde que 
atuam na APS no município de Itaguaí. O objetivo do pré-teste foi de levantar as necessidades de alterações listando as observações e sugestões dos profissionais avaliadores. O arquivo de instalação foi enviado por e-mail aos participantes e os mesmos retornaram o TCLE e o questionário por meio eletrônico para a pesquisadora. Os critérios utilizados para avaliação do aplicativo foram a ergonomia e a usabilidade. Os 2 critérios perfazem um total possível de 90 pontos. Utilizou-se a escala de likert de 5 pontos em que a menor pontuação se equivaleu a avaliação "discordo plenamente - 1 ponto" e a maior pontuação a "concordo plenamente - 5 pontos". Denominamos os avaliadores por algarismos arábicos de 1 a 20.

Em relação ao critério ergonomia, a organização obteve pontuação total pelos avaliadores $4,5,6,7,8,9,10,11,12,13,14,15,16,17,18,19$ e 20 - 10 pontos "concordo plenamente". Já a interface, obteve pontuação total - 20 pontos pelos avaliadores 1, 4, 5, 6, 7, 12, 13, 14, 15, 16, 17, 18, 19 e 20. Quanto ao conteúdo do aplicativo referente às informações apresentadas e sua interrelação com a área em questão, os avaliadores $5,6,7,8,9,10,11,12,13,14,15,16,17,18,19$ e 20 pontuaram com a nota total - 15 pontos. Quanto à parte técnica, os avaliadores1, 4, 5, $6,7,8,9,10,11,12,13,14,15,16,17,18,19$ e 20 pontuaram com a nota máxima - 10 pontos. O critério usabilidade obteve 35 pontos de pontuação total pelos avaliadores 4 , $5,6,7,8,9,11,12,14,15,16,17,18,19$ e 20.

No critério Organização, 17 avaliadores pontuaram com nota máxima ficando o quesito com média total de 4,92. No Critério Interface, 14 avaliadores deram a nota máxima ficando o quesito com 4,82 de média geral. Já o critério conteúdo recebeu nota máxima de 16 avaliadores ficando com média geral 4,93. O critério técnica, ficou com média geral de 4,93, tendo recebido nota máxima de 18 avaliadores. O critério usabilidade ficou com média geral de 4,89, tendo recebido nota máxima de 15 avaliadores. Ao final do pré-teste, concluiu-se que os profissionais avaliaram positivamente o aplicativo tanto em relação à sua ergonomia quanto em relação à sua usabilidade.

\section{Discussão}

Atualmente, a utilização de tecnologia digital para cuidados em saúde constitui um importante recurso para auxiliar profissionais e pacientes. Curioni, Brito e Boccolini argumentam que esse tipo de suporte vem se tornando uma potencial ferramenta nas práticas médicas e de saúde pública, na medida em que colaboram para a melhoria da gestão da informação, o acesso aos serviços, a qualidade do cuidado prestado e a contenção de custos. (CURIONI,2003) Quando é direcionado para profissionais de saúde a criação de aplicativos com temática voltada para a saúde pode contemplar 
médicos, dentistas, fisioterapeutas, fonoaudiólogos, cuidadores e outros grupos, compartilhando áreas e conhecimentos específicos. (OEHLER,2010)

O aplicativo "Hemoapp", assim denominado pelos autores deste trabalho, se configura como um aplicativo que foi desenvolvido com o objetivo principal de auxiliar os profissionais de saúde no manejo do paciente portador de Hemofilia e Doença de Von Willebrand. O aplicativo tem a possibilidade de ser utilizado como ferramenta de apoio ao tratamento do paciente portador de coagulopatias hereditárias na APS. Além de sua utilização no ambiente físico do serviço de saúde, por ter sido desenvolvido para dispositivos móveis, o aplicativo pode ser utilizado como ferramenta para avaliação dos pacientes em visitas domiciliares. Diante das funcionalidades do aplicativo desenvolvido foi necessário verificar sua utilização na prática clínica, para isso foi realizado um préteste com profissionais de saúde. É importante ressaltar que alterações e correções visando à atualização do sistema de acordo com novas determinações no tratamento de pacientes com coagulopatias hereditárias sempre deverão ser consideradas, uma vez que o desenvolvimento de um software para a saúde é um processo dinâmico (BARRA, 2008).

\section{Conclusão}

Devido ao aumento no número de brasileiros portadores de coagulopatias hereditárias e suas dificuldades em conseguir atendimento na atenção básica em saúde, visando melhorar o acolhimento desse paciente, corroboram, a importância da criação de um aplicativo que auxilie o profissional na classificação, diagnóstico e tratamento dos pacientes portadores de coagulopatias hereditárias. Acreditamos que a elaboração de um aplicativo para dispositivos móveis possibilitaria não só o suporte com evidências científicas para avaliação e classificação do paciente portador de coagulopatias hereditárias, como também a capacitação dos profissionais no cuidado, o que permitiria um gerenciamento de qualidade do atendimento desses pacientes na atenção primária. O presente estudo se propôs a elaborar um aplicativo que desse suporte à decisão clínica, como também capacitasse os profissionais de saúde no manejo do paciente, possibilitando um melhor gerenciamento dos pacientes com coagulopatias hereditárias. Assim, foi desenvolvido um aplicativo para dispositivos móveis na plataforma Android. $O$ profissional de saúde, após cadastro, tem acesso a dois módulos principais, um módulo de hemofilia e um para a Doença de Von Willebrand. Após elaboração do aplicativo, realizou-se um pré-teste com profissionais de saúde da APS a fim de avaliar sua ergonomia, usabilidade e levantar possíveis modificações a serem realizadas no aplicativo e nos questionários de avaliação. Por meio do pré-teste pudemos verificar também a necessidade contínua de atualização profissional e a incorporação de novas 
tecnologias no cuidado, que acabam por facilitar e agilizar a assistência do paciente. Apesar das limitações encontradas durante o desenvolvimento do estudo, considerandose a fase de desenvolvimento do aplicativo, uma vez que a pesquisadora não dispunham do embasamento necessário para a programação computacional. $\mathrm{E}$ em relação a realização do pré-teste, a dificuldade encontrada foi em conseguir resposta dos avaliadores, foi necessário a realização de diversos contatos via e-mail e telefônico para que os avaliadores respondessem o questionário e preenchessem o termo de livre esclarecimento. Verifica-se que a utilização de novas tecnologias no acompanhamento de pacientes portadores de coagulopatias é completamente possível.

\section{Bibliografia}

BARRA, D C C. Processo de enfermagem informatizado em terapia intensiva em ambiente PDA (Personal Digital Assistant) a partir da CIPE ${ }^{\circledR}$ versão 1.0, 2008 [Dissertação] / Daniela Couto Carvalho Barra - Florianópolis (SC): UFSC/PEN, 2008.

BRASIL. Ministério da Saúde. Secretaria de Atenção à Saúde. Coordenação?Geral de Sangue e Hemoderivados. Perfil das coagulopatias hereditárias no Brasil : 2014 / Ministério da Saúde, Secretaria de Atenção à Saúde, Coordenação?Geral de Sangue e Hemoderivados. - Brasília : Ministério da Saúde, 2015b. 62 p. il

CHAUDHRY B, WANG J, WU S, MAGLIONE M, MOJICA W, ROTH E, et al. Systematic review: impact of health information technology on quality, efficiency, and costs of medical care. Ann Intern Med 2006; 144(10):742-52

COCKBURN, A. e HIGHSMITH, J. "Agile Software Development: The Business of Innovation", IEEE Computer, Sept.2001, p. 120-122

CONSTANTINOU,A. , CAMILLERI, E., KAPETANASKIS, M. Mobile developer economics 2010 and beyond. 2010

CURIONI CC, BRITO FDSB, BOCCOLINI CS. O uso de tecnologias de informação e comunicação na área da Nutrição. Jornal Brasileiro de Telessaúde. 2013; 2(3): 51-59.

FEDERAÇÃO BRASILEIRA DE HEMOFILIA. Convivendo com a Hemofilia: manual de bolso. Disponível em: . Acesso em: 19 jun. 2016.

FIGUEIREDO CMS, NAKAMURA E. Computação móvel: novas oportunidades e desafios. Rev T\&C Amazonia. 2003; 1(2):16-28. 
FLANAGAN, David; FERGUSON, Paula (2002). JavaScript: The Definitive Guide 4th ed. [S.I.]: O'Reilly \& Associates.

GARG AX, ADHKARI NKJ, MCDONALD H, ROSAS-ARELLANO MP, DEVEREAUX PJ, $B E Y E N E$ J, et. al. Effects of computerized clinical decision support systems on practitioner performance and patient outcomes. JAMA 2005; 293(10):1223-38

LORENZI TF — Patologia da Hemostasia, em: D'amico E, Daniel MM, Silveira PAA — Manual de Hematologia Propedêutica e Clínica, $3^{\mathrm{a}} \mathrm{Ed}$, Rio de Janeiro, Medsi, 2003;477-552.

KUKAFKA R. Information technology, education and health care: constructivism in the 21st century. Educ Stud 2001;27(3):229-35

OEHLER RL, SMITH K, TONEY JF: Infectious diseases resources for iphone. Clin Infect Dis 2010, 50:1268-1274

PETROVITCH CT, DRUMMOND JC - Hemoterapia e Hemostasia, em: BARASH PG, CULlen BF, StOelting RK - Anestesia Clínica, 4aㅡ Ed,São Paulo, Manole, 2004;201-238.

SOTO RG, CHU LF, GOLDMAN JM, RAMPIL IJ, RUSKIN KJ. Communicatin in critical care environments: mobile telephones improve patient care. Anest analg 2006; 102(2):534-41

STARDUST. Mobile application \&reputation. Stardust, 2013. Disponível em: http://www.stardust-testing.com/ressources_1/notice-m-reputation-en.pdf Acesso em: 05 de novembro de 2016 